\title{
Simultaneous identification of molecular and mating types within the Cryptococcus species complex by PCR-RFLP analysis
}

Correspondence

Zhirong Yao

zryaosmu@sohu.com

Received 21 May 2008

Accepted 29 July 2008

\author{
Xiaobo Feng, ${ }^{1}$ Zhirong Yao, ${ }^{1}$ Daming Ren $^{2}$ and Wanqing Liao ${ }^{3}$ \\ ${ }^{1}$ Medical Mycology Laboratory, Xinhua Hospital, Shanghai Jiaotong University School of Medicine,
Shanghai, PR China \\ ${ }^{2}$ State Key Laboratory of Genetic Engineering, Institute of Genetics, Fudan University, Shanghai, \\ PR China \\ ${ }^{3}$ Medical Mycology Laboratory, Shanghai Changzheng Hospital, Shanghai, PR China
}

\section{INTRODUCTION}

The Cryptococcus species complex contains the most common causative agents of fungal meningoencephalitis in immunocompromised patients, particularly in the AIDS population (Mitchell \& Perfect, 1995; Casadevall \& Perfect, 1998). Within the Cryptococcus species complex, two species, Cryptococcus neoformans and Cryptococcus gattii, have been named. Both species have a bipolar mating system involving $\alpha$ and a mating types (MAT) (KwonChung et al., 2002; Kwon-Chung \& Varma, 2006). The Cryptococcus species complex is currently divided into eight major molecular types: VNI/AFLP1 and VNII/AFLP1A/1B (C. neoformans var. grubii, serotype A); VNIII/AFLP3 (AD hybrid, serotype AD); VNIV/AFLP2 (C. neoformans var. neoformans, serotype D); and VGI/AFLP4, VGII/AFLP6, VGIII/AFLP5 and VGIV/AFLP7 (C. gattii, serotypes B and C) (Boekhout et al., 2001; Latouche et al., 2003; Meyer et al., 2003; Bovers et al., 2008a). In addition to the eight established molecular types with opposite mating types, a genotype, $\mathrm{VNB}$, unique to Botswana and a few novel

The GenBank/EMBL/DDBJ accession numbers for the CAP1 and GEF1 sequences for the reference strains of WM626, CBS1930, WM161, B4546 and WM779 are EU571727-EU571731 and EU571732-EU571736, respectively. hybrids of $\mathrm{A} \alpha \mathrm{D} \alpha$ and $C$. neoformans $\times C$. gattii have been described in recent studies (Litvintseva et al., 2005a, 2006; Bovers et al., 2006; Lin et al., 2007; Bovers et al., 2008b). Differences in biology, epidemiology, pathogenicity, clinical features and drug susceptibility have been reported to be associated with species, varieties, molecular types and mating types within the Cryptococcus species complex (Kwon-Chung et al., 1992; Speed \& Dunt, 1995; Casadevall \& Perfect, 1998; Meyer et al., 2003; Trilles et al., 2004; Campbell et al., 2005; Fraser et al., 2005).

C. neoformans is a ubiquitous pathogen associated with avian faeces and decaying wood, being responsible for most cases of cryptococcosis. The most common variety, $C$. neoformans var. grubii, primarily infects immunocompromised individuals and accounts for most cryptococcal infections ( $>90 \%$ ) (Casadevall \& Perfect, 1998). Strains of

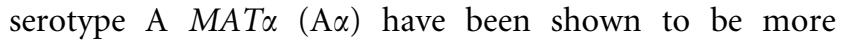
pathogenic than Aa strains in several reports (Lengeler et al., 2000; Keller et al., 2003; Barchiesi et al., 2005). Despite molecular types VNI and VNII both appearing to be distributed globally, VNII is recovered less frequently (Meyer et al., 2003). In addition, a unique genotype, VNB, belonging to C. neoformans var. grubii has recently been found to be restricted to Botswana. VNB includes a 
large proportion of fertile isolates of MATa (Litvintseva et al., 2006). The second variety, C. neoformans var. neoformans, causing cases mainly in Europe, has been reported to be less virulent and more susceptible to fluconazole than $C$. neoformans var. grubii (Tortorano et al., 1997; Casadevall \& Perfect, 1998). In murine models, $\mathrm{D} \alpha$ strains are more virulent than congenic Da strains (Kwon-Chung et al., 1992). AD strains are hybrids between the two varieties, and possess two mating type alleles with one from each of the varieties. It is suggested that $\mathrm{AD}$ hybrids may be more common in clinical and environmental sources than was previously thought (Litvintseva et al., 2005a; Viviani et al., 2006). Further studies have shown that AD hybrids exhibit hybrid vigour in the laboratory (Lin et al., 2007; Litvintseva et al., 2007). Interestingly, strains of $\mathrm{A} \alpha \mathrm{Da}$ have been reported to be more virulent than $\mathrm{AaD} \alpha$ strains (Barchiesi et al., 2005). Moreover, a hybrid genotype of $\mathrm{A} \alpha \mathrm{D} \alpha$ has recently been recovered from the USA and Italy, implicating same-sex mating between $\alpha$ mating types within this species (Litvintseva et al., 2005a; Lin et al., 2007).

C. gattii, previously known as C. neoformans var. gattii, has been raised to species level (Kwon-Chung et al., 2002). This sibling species was primarily restricted to tropical and subtropical climates until an outbreak on Vancouver Island, Canada, in 2000. It inhabits decaying wood of eucalypts or other tree species and primarily infects immunocompetent individuals (Casadevall \& Perfect, 1998; Kidd et al., 2004). Compared with C. neoformans, the clinical characteristics of C. gattii have been reported to be different. It is the cause of cryptococcomas and has a lower susceptibility to several antifungal agents, a need for prolonged treatment and a higher mortality rate (Speed \& Dunt, 1995; Trilles et al., 2004). Like C. neoformans, isolates of C. gattii with the MATa mating type are also predominant in clinical and environmental samples, especially in the former (Campbell et al., 2005; Fraser et al., 2005; Litvintseva et al., 2005b). Among the four previously defined molecular types of $C$. gattii, molecular types VGI and VGII are widely distributed, whereas VGIII and VGIV are usually isolated from tropical climates (Meyer et al., 2003; Litvintseva et al., 2005b). Strains of VGII MAT $\alpha$ have been revealed to be related to a high incidence of C. gattii infections, such as the cryptococcosis outbreak on Vancouver Island (Kidd et al., 2004; Campbell et al., 2005; Fraser et al., 2005). Strains of VGIV MATa have been recovered mainly from patients with AIDS in sub-Saharan Africa (Litvintseva et al., 2005b). Furthermore, several novel C. gattii $\times$. neoformans hybrids in which the $C$. gattii alleles originated from the molecular type VGI have been described in clinical samples (Bovers et al., 2006, 2008b).

The population structure of the eight molecular types has been confirmed by sequence and phylogenetic analyses of multiple genes (Fraser et al., 2005; Bovers et al., 2008a). RFLP analysis based on a single gene was developed for the differentiation of the molecular types and confirmed to be a reliable and easy-to-use method (Latouche et al., 2003;
Meyer et al., 2003). In a recent study, sequence analysis of the CAP1 gene, which is located at the MAT locus, indicated that sequence variation due to the mating type was greater than that due to the molecular type divergence. The dendrogram showed that strains clustered according to mating type, and in each of the two clades, strains clustered according to molecular type (Kidd et al., 2005). In a previous study by us, partial sequences of the GEF1 gene (a gene also located at the MAT locus) for strains representative of the distinct molecular and mating types were determined. The results showed that this gene exhibited genetic traits similar to those of the CAP1 gene (X. Feng and others, unpublished results). These data demonstrated that the molecular and mating types of strains belonging to the Cryptococcus species complex could be determined simultaneously by sequencing either of the two genes.

In the present study, two alternative approaches using PCRRFLP analysis, based on the CAP1 and GEF1 genes, requiring only common materials in the laboratory were developed to determine simultaneously the molecular and mating types of isolates of the Cryptococcus species complex.

\section{METHODS}

Strains and DNA extraction. One hundred and forty-four isolates of the Cryptococcus species complex comprising 96 C. neoformans isolates and 48 C. gattii isolates were used in this study. Among the tested strains, 135 represented the 14 distinct groups of the molecular

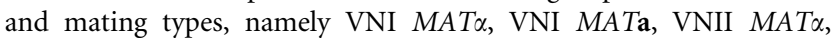
VNIII MAT $\alpha / \mathbf{a}$, VNIII MATa/ $\alpha$, VNIV MAT $\alpha$, VNIV MATa, VGI $M A T \alpha$, VGI MATa, VGII MAT $\alpha$, VGII MATa, VGIII MAT $\alpha$, VGIII $M A T \mathbf{a}$ and VGIV MAT $\alpha$. The other nine isolates represented the rare subtypes of the genotype VNB (including MAT $\alpha$ and MATa) and the $\mathrm{AD}$ hybrid of $\mathrm{A} \alpha \mathrm{D} \alpha$. The data for the strains tested are shown in Table 1. The molecular and mating types of 69 isolates were determined previously in other studies, and those of the other 75 isolates (Table 1) were determined by PCR fingerprinting (Meyer et al., 2003) and PCR analysis with primers specific for the STE20 and STE12 genes situated within the MAT locus as described previously by Lengeler et al. (2001) and Fraser et al. (2003), respectively. The strains recovered from China were identified as Cryptococcus species by morphology and biochemical and physiological tests, such as microscopic observation of India ink preparation, black or brown colony appearance on L-3,4-dihydroxyphenylalanine agar, a positive urease reaction and the ability to grow at $37{ }^{\circ} \mathrm{C}$ (X. Feng and others, unpublished results). Strains were maintained on yeast extract-peptone-glucose medium at $30{ }^{\circ} \mathrm{C}$ for 2 days before testing. Genomic DNA was prepared from each strain as described previously (Meyer et al., 2003).

PCR amplification. The sequences of the CAP1 and GEF1 genes from the reference strains representing distinct molecular and mating types, including H99 (VNI MAT $\alpha$; GenBank accession no. AF542529), 125.91 (VNI MATa; AF542528), JEC21 (VNIV MAT $\alpha$; AF542531), JEC20 (VNIV MATa; AF542530), WM276 (VGI MAT $\alpha$; AY710430), E566 (VGI MATa; AY710429) and R265 (VGII MAT $\alpha$ DQ096809), were obtained from GenBank. The sequences for the reference strains of WM626 (VNII MAT $\alpha$ ), CBS1930 (VGII MATa), WM161 (VGIII $M A T \alpha$ ), B4546 (VGIII MATa) and WM779 (VGIV MAT $\alpha$ ) were generated in our study using TA cloning and a nested sequencing approach. Primers were designed from the consensus sequence based on alignment of the targeted gene sequences from the above reference strains. For the CAP1 gene, two primers (CAP1F, 5' - 
Table 1. Strains of the Cryptococcus species complex tested in this study and RFLP results obtained for the CAP1 and GEF1 genes

\begin{tabular}{|c|c|c|c|c|c|c|c|}
\hline Strain & Origin ${ }^{\star}$ & Serotype & $\begin{array}{l}\text { Molecular } \\
\text { type }\end{array}$ & $\begin{array}{l}\text { Mating } \\
\text { type }\end{array}$ & CAP1 & GEF1 & Reference/source \\
\hline \multicolumn{8}{|c|}{$\begin{array}{l}\text { C. neoformans } \\
\text { var. grubii }\end{array}$} \\
\hline H99 & CSF, USA & A & VNI & $\alpha$ & $\mathrm{C} 1$ & G1 & Lengeler et al. (2001); J. R. Perfect \\
\hline RV45886 & Belgium & A & VNI & $\alpha$ & $\mathrm{C} 1$ & G1 & $\dagger ;$ C. De Vroey \\
\hline RV67754 & Belgium & A & VNI & $\alpha$ & $\mathrm{C} 1$ & G1 & This study; C. De Vroey \\
\hline RV67746 & Belgium & A & VNI & $\alpha$ & $\mathrm{C} 1$ & G1 & This study; C. De Vroey \\
\hline XH95 & Clinical, China & $\mathrm{A}$ & VNI & $\alpha$ & $\mathrm{C} 1$ & G1 & $\dagger$ \\
\hline XH109 & Clinical, China & A & VNI & $\alpha$ & $\mathrm{C} 1$ & G1 & $\dagger$ \\
\hline XH89 & Clinical, China & A & VNI & $\alpha$ & $\mathrm{C} 1$ & G1 & $\dagger$ \\
\hline XH37 & Clinical, China & A & VNI & $\alpha$ & $\mathrm{C} 1$ & G1 & $\dagger$ \\
\hline XH102 & Clinical, China & A & VNI & $\alpha$ & $\mathrm{C} 1$ & G1 & $\dagger$ \\
\hline XH81 & Clinical, China & A & VNI & $\alpha$ & $\mathrm{C} 1$ & G1 & $\dagger$ \\
\hline XH116 & Clinical, China & A & VNI & $\alpha$ & $\mathrm{C} 1$ & G1 & $\dagger$ \\
\hline XH99 & Clinical, China & A & VNI & $\alpha$ & $\mathrm{C} 1$ & G1 & $\dagger$ \\
\hline XH83 & Clinical, China & A & VNI & $\alpha$ & $\mathrm{C} 1$ & G1 & $\dagger$ \\
\hline XH103 & Clinical, China & $\mathrm{A}$ & VNI & $\alpha$ & $\mathrm{C} 1$ & G1 & $\dagger$ \\
\hline XH113 & Clinical, China & A & VNI & $\alpha$ & $\mathrm{C} 1$ & G1 & $\dagger$ \\
\hline XH114 & Clinical, China & A & VNI & $\alpha$ & $\mathrm{C} 1$ & G1 & $\dagger$ \\
\hline XH22 & Clinical, China & A & VNI & $\alpha$ & $\mathrm{C} 1$ & G1 & $\dagger$ \\
\hline XH110 & Clinical, China & A & VNI & $\alpha$ & $\mathrm{C} 1$ & G1 & $\dagger$ \\
\hline XH39 & Clinical, China & A & VNI & $\alpha$ & $\mathrm{C} 1$ & G1 & $\dagger$ \\
\hline XH55 & Clinical, China & A & VNI & $\alpha$ & $\mathrm{C} 1$ & G1 & $\dagger$ \\
\hline XH106 & Clinical, China & A & VNI & $\alpha$ & $\mathrm{C} 1$ & G1 & $\dagger$ \\
\hline XH107 & Clinical, China & $\mathrm{A}$ & VNI & $\alpha$ & $\mathrm{C} 1$ & G1 & $\dagger$ \\
\hline XH104 & Clinical, China & A & VNI & $\alpha$ & $\mathrm{C} 1$ & G1 & $\dagger$ \\
\hline XH21 & Clinical, China & A & VNI & $\alpha$ & $\mathrm{C} 1$ & G1 & $\dagger$ \\
\hline XH28 & Clinical, China & A & VNI & $\alpha$ & $\mathrm{C} 1$ & G1 & $\dagger$ \\
\hline XH7 & Clinical, China & A & VNI & $\alpha$ & $\mathrm{C} 1$ & G1 & $\dagger$ \\
\hline XH70 & Clinical, China & A & VNI & $\alpha$ & $\mathrm{C} 1$ & G1 & $\dagger$ \\
\hline XH68 & Clinical, China & A & VNI & $\alpha$ & $\mathrm{C} 1$ & G1 & $\dagger$ \\
\hline XH85 & Clinical, China & A & VNI & $\alpha$ & $\mathrm{C} 1$ & G1 & $\dagger$ \\
\hline XH82 & Clinical, China & A & VNI & $\alpha$ & $\mathrm{C} 1$ & G1 & $\dagger$ \\
\hline XH65 & Clinical, China & A & VNI & $\alpha$ & $\mathrm{C} 1$ & G1 & $\dagger$ \\
\hline XH76 & Clinical, China & A & VNI & $\alpha$ & $\mathrm{C} 1$ & G1 & $\dagger$ \\
\hline XH92 & Clinical, China & $\mathrm{A}$ & VNI & $\alpha$ & $\mathrm{C} 1$ & G1 & $\dagger$ \\
\hline XH59 & Clinical, China & A & VNI & $\alpha$ & $\mathrm{C} 1$ & G1 & $\dagger$ \\
\hline XH43 & Clinical, China & $\mathrm{A}$ & VNI & $\alpha$ & $\mathrm{C} 1$ & G1 & $\dagger$ \\
\hline $\mathrm{XH} 42$ & Clinical, China & A & VNI & $\alpha$ & $\mathrm{C} 1$ & G1 & $\dagger$ \\
\hline XH79 & Clinical, China & A & VNI & $\alpha$ & $\mathrm{C} 1$ & G1 & $\dagger$ \\
\hline XH87 & Clinical, China & A & VNI & $\alpha$ & $\mathrm{C} 1$ & G1 & $\dagger$ \\
\hline
\end{tabular}


Table 1. cont.

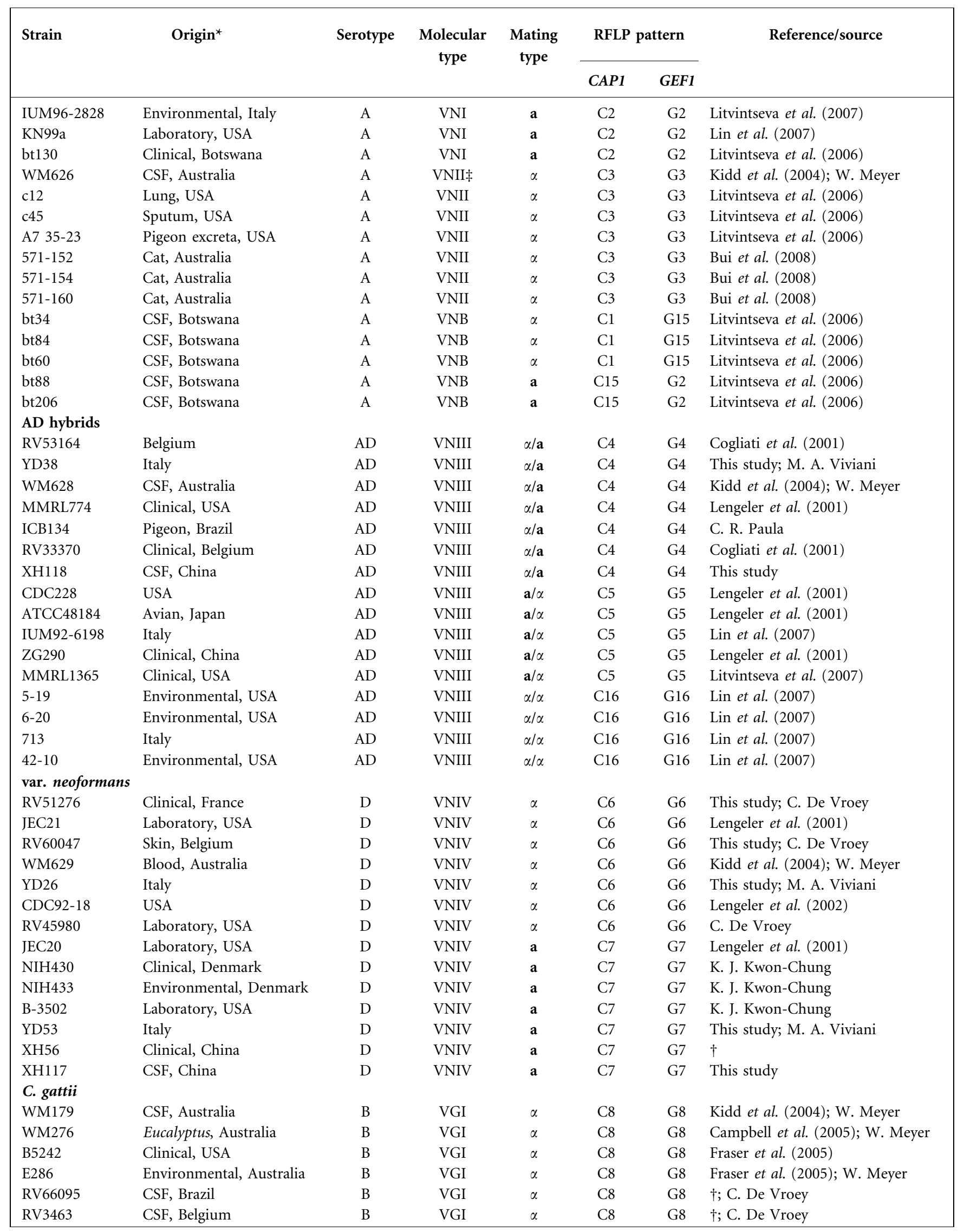


Table 1. cont.

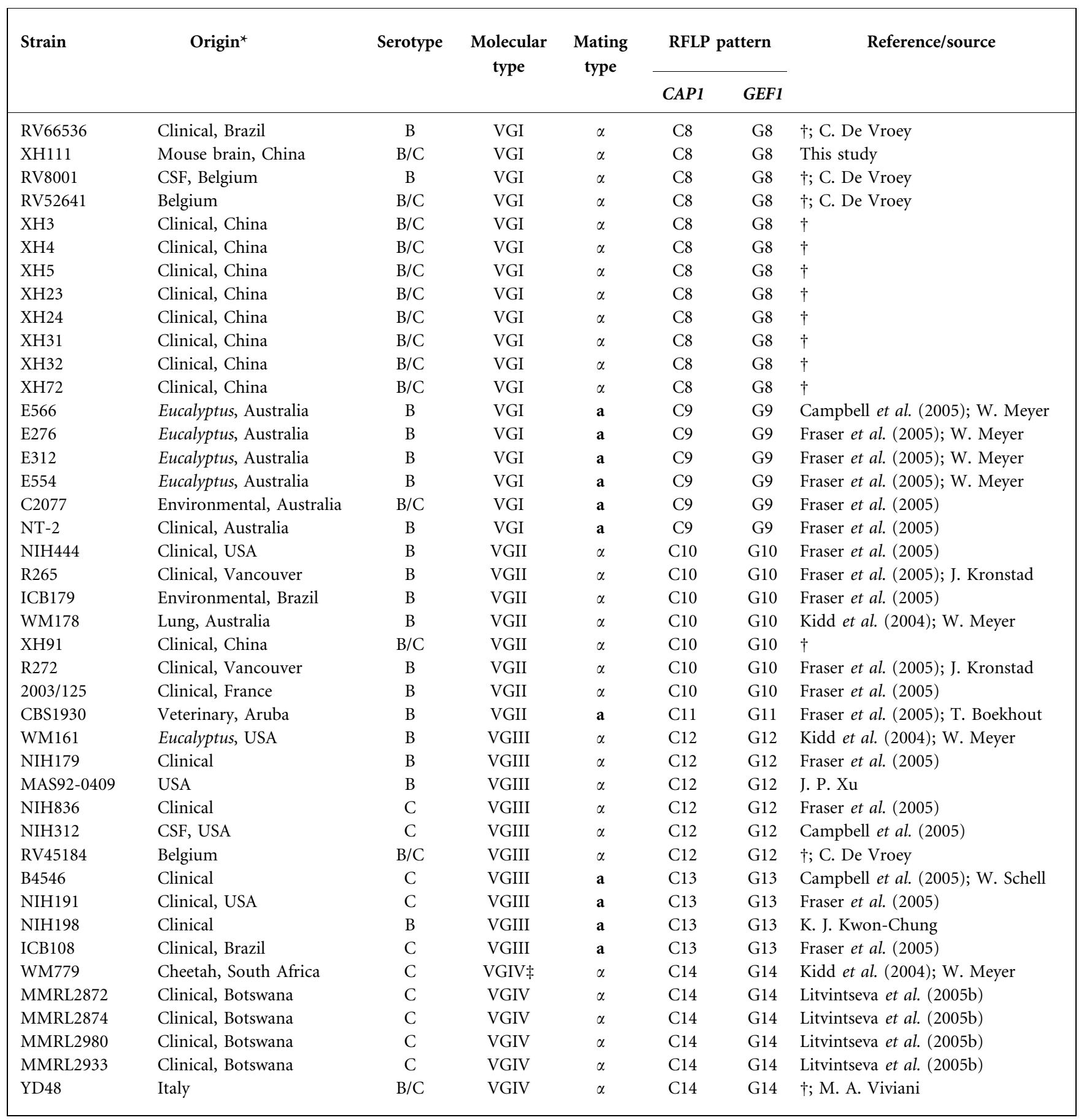

${ }^{*} \mathrm{CSF}$, cerebrospinal fluid.

$\dagger$ The molecular types and/or mating types were determined in a previous study by us by PCR fingerprinting and/or PCR analysis of the STE20 and STE12 genes, respectively (X. Feng and others, unpublished results).

\$No mating type a within these molecular types has been reported until now.

TCAGATCAACCGCGACATCG-3'; and CAP1R, 5' ${ }^{\prime}$-TGAACCCAAGGGGTAAGCCA-3') comprising nt 664-683 and nt 1879-1898, respectively, based on the CAP1 sequence of strain JEC21 were designed to amplify a portion of the CAP1 gene from all strains investigated (Fig. 1a). For the GEF1 gene, two primers (GEF1F, 5' -
GGACCCATGCCTGAAATGTG-3'; and GEF1R, 5'-TACGCTTGCCCCGATCTG-3') comprising nt 1703-1722 and nt 3024-3041, respectively, based on the GEF1 sequence of the strain JEC21 were designed to amplify a portion of the GEF1 gene from all strains tested (Fig. 1b). 
(a)

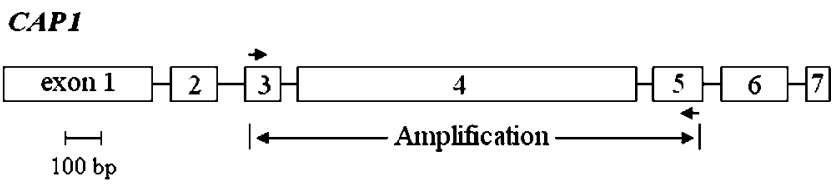

(b)

GEF1

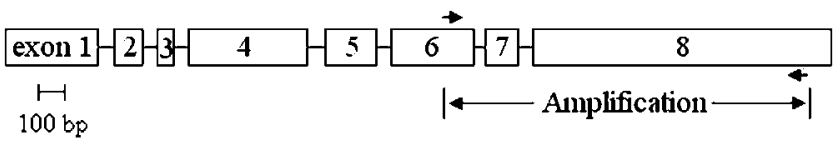

Fig. 1. Schematic representation of the amplified regions of the CAP1 gene (a) and GEF1 gene (b). Primer binding sites are shown by short arrows. Open boxes represent exons, with the order indicated by numbers, whilst lines between exons represent introns.

Amplification was performed in $1 \times$ PCR buffer containing $1.5 \mathrm{mM}$ $\mathrm{MgCl}_{2}, 0.2 \mathrm{mM}$ each $\mathrm{dNTP}, 10 \mu \mathrm{M}$ each primer and $1.5 \mathrm{U}$ Taq polymerase. The PCR conditions for both amplifications were: initial denaturation at $94{ }^{\circ} \mathrm{C}$ for $5 \mathrm{~min}$, followed by 31 cycles of $94{ }^{\circ} \mathrm{C}$ for $30 \mathrm{~s}$, $57{ }^{\circ} \mathrm{C}$ for $30 \mathrm{~s}$ and $72{ }^{\circ} \mathrm{C}$ for $1.2 \mathrm{~min}$, with a final extension step at $72{ }^{\circ} \mathrm{C}$ for $5 \mathrm{~min}$. PCR products were analysed on a $1 \%(\mathrm{w} / \mathrm{v})$ agarose gel.

RFLP analysis. Using DNAMAN 5.2.2 software, analyses of sequence comparisons, restriction endonuclease selection, restriction maps and unique cutting sites were conducted based on the amplified DNA sequences of the CAP1 and GEF1 genes from type strains H99, 125.91, WM626, JEC21, JEC20, WM276, E566, R265, CBS1930, WM161, B4546 and WM779, representing 12 distinct groups of molecular and mating types. Additionally, $\mathrm{AD}$ hybrids were analysed using a combination of serotype A (VNI) and serotype D (VNIV) alleles. The restriction endonucleases AvaI, HgiCI, EcoRII and PstI were chosen and predicted to yield specific RFLP patterns for the differentiation of the distinct groups of all molecular and mating types following digestion of the PCR products from the CAP1 gene. The PCR products were subjected to separate restriction digestions with AvaI and HgiCI enzymes in a first-line identification; PCR products with patterns identical to those of molecular type VNI or VNII (both MATa) were further digested with EcoRII, and PCR products with patterns identical to those of molecular type VGIII or VGIV (both MAT $\alpha$ ) were further digested with PstI. The restriction endonucleases EcoT14I and HapII were selected and predicted to yield specific RFLP patterns for the differentiation of the distinct groups of all molecular and mating types following digestion of the PCR products from the GEF1 gene. The PCR products were subjected to separate restriction digestions with EcoT14I and HapII enzymes for identification.

All restriction digestions were performed according to the manufacturer's instructions (TaKaRa). The reaction mixture was incubated at $37{ }^{\circ} \mathrm{C}$ for $3 \mathrm{~h}$ before separation on a $2.0 \%(\mathrm{w} / \mathrm{v})$ agarose gel at $100 \mathrm{~V}$ for $1 \mathrm{~h}$ and then visualization under UV light.

\section{RESULTS AND DISCUSSION}

\section{RFLP identification of the molecular and mating types}

The 144 C. neoformans and C. gattii isolates tested gave positive amplification with both primer pairs: CAP1F/
CAP1R and GEF1F/GEF1R. For all strains assayed, a fragment of approximately $1235 \mathrm{bp}$ was amplified from the genomic DNA with primers CAP1F and CAP1R, and one of approximately $1339 \mathrm{bp}$ with primers GEF1F and GEF1R.

By separate digestions with AvaI and HgiCI, RFLP analysis of the fragments amplified from the CAP1 gene generated 12 different patterns, compatible with the distinct groups of the eight molecular types (VNI, VNII, VNIII, VNIV, VGI, VGII, VGIII and VGIV) and the two mating types ( $\alpha$ and a) within the 135 isolates of the Cryptococcus species complex. The same restriction pattern was shown by the molecular types VNI and VNII (both MAT $\alpha$ ) and a different pattern was shown by both VGIII and VGIV (both $M A T \alpha$ ), as predicted by in silico analysis. In concordance with the in silico analysis, molecular types VNI and VNII (both MAT $\alpha$ ) could be differentiated further by EcoRII digestion, and VGIII and VGIV (both MAT $\alpha$ ) could be differentiated further by Pst digestion (Tables 1 and 2; Fig. 2). RFLP analysis of the fragments amplified from the GEF1 gene via separate digestions with EcoT14I and HapII generated 14 different patterns corresponding exactly to the distinct groups of the eight molecular types and the two mating types for the 135 isolates as expected from the in silico analysis (Tables 1 and 2; Fig. 3). Thus the 135 cryptococcal isolates were successfully grouped corresponding to their unique molecular and mating types using either of the two PCR-RFLP approaches. No profile variation was observed when the patterns were analysed, confirming the reliability of the two typing approaches. In addition, the unusual $\mathrm{AD}$ hybrid of $\mathrm{A} \alpha \mathrm{D} \alpha$ was also distinguished by the established RFLP patterns by either of the two typing approaches (Tables 1 and 2; Figs 4 and 5).

\section{Identification of VNB strains}

The ability of the two RFLP typing approaches to identify the rare genotype $\mathrm{VNB}$ and its mating type was also investigated. The VNB MAT $\alpha$ strains exhibited the same restriction profile as the molecular type VNI MAT $\alpha$ isolates after separate digestions of the CAP1 gene with AvaI, HgiCI and EcoRII, whereas the VNB MATa strains showed a unique pattern distinct from the 12 patterns established by separate digestions with AvaI and HgiCI (Tables 1 and 2; Fig. 6). The VNB MATa strains displayed a unique pattern in comparison with all patterns established for the GEF1 gene (Tables 1 and 2; Fig. 5), but the restriction pattern obtained from the VNB MATa strains was identical to the pattern of the molecular type VNI MATa strains. These results suggest that VNB MAT $\alpha$ and VNB MATa strains could not both be distinguished from VNI strains by either of the two methods. Therefore, a combination of the two typing approaches is required for the identification of the VNB strains.

\section{Analysis of AD hybrid subtypes}

Multilocus genotyping methods such as PCR fingerprinting, amplified fragment length polymorphism analysis and 
Table 2. Categories of restriction profiles from the CAP1 and GEF1 gene fragments with the corresponding molecular and mating types

\begin{tabular}{|c|c|c|c|c|c|c|c|c|}
\hline \multirow{2}{*}{$\begin{array}{l}\text { Molecular and } \\
\text { mating types }\end{array}$} & \multirow[t]{2}{*}{ RFLP pattern } & \multicolumn{4}{|c|}{ Profile $(C A P 1)^{\star}$} & \multirow[t]{2}{*}{ RFLP pattern } & \multicolumn{2}{|c|}{ Profile $(G E F 1)^{*}$} \\
\hline & & AvaI & HgiCI & EcoRII & Pst $\mathrm{I}$ & & EcoT14I & HapII \\
\hline VNI $M A T \alpha$ & $\mathrm{C} 1$ & A1 & $\mathrm{H} 1$ & E1 & $\mathrm{ND}$ & G1 & Ec1 & Hal \\
\hline VNI MATa & $\mathrm{C} 2$ & $\mathrm{~A} 2$ & $\mathrm{H} 2$ & ND & ND & $\mathrm{G} 2$ & Ec2 & Ha2 \\
\hline VNII $M A T \alpha$ & $\mathrm{C} 3$ & $\mathrm{~A} 1$ & $\mathrm{H} 1$ & E2 & $\mathrm{ND}$ & G3 & $\mathrm{Ec1}$ & Ha3 \\
\hline VNIV $M A T \alpha$ & $\mathrm{C} 6$ & A5 & $\mathrm{H} 1$ & $\mathrm{ND}$ & $\mathrm{ND}$ & G6 & Ec5 & Ha6 \\
\hline VNIV MATa & $\mathrm{C} 7$ & A6 & $\mathrm{H} 1$ & $\mathrm{ND}$ & $\mathrm{ND}$ & G7 & Ec2 & Ha6 \\
\hline VGI $M A T \alpha$ & $\mathrm{C} 8$ & A7 & $\mathrm{H} 4$ & $\mathrm{ND}$ & $\mathrm{ND}$ & G8 & Ec6 & Ha7 \\
\hline VGI MATa & $\mathrm{C} 9$ & $\mathrm{~A} 2$ & H5 & $\mathrm{ND}$ & $\mathrm{ND}$ & G9 & Ec7 & Ha8 \\
\hline VGII $M A T \alpha$ & $\mathrm{C} 10$ & A8 & $\mathrm{H} 4$ & ND & $\mathrm{ND}$ & G10 & Ec5 & Ha9 \\
\hline VGII MATa & $\mathrm{C} 11$ & $\mathrm{~A} 2$ & H6 & $\mathrm{ND}$ & $\mathrm{ND}$ & G11 & Ec5 & Ha10 \\
\hline VNB $M A T \alpha$ & $\mathrm{C} 1$ & A1 & $\mathrm{H} 1$ & E1 & $\mathrm{ND}$ & G15 & Ec1 & Ha8 \\
\hline VNB MATa & $\mathrm{C} 15$ & A6 & $\mathrm{H} 2$ & ND & $\mathrm{ND}$ & G2 & $\mathrm{Ec} 2$ & Ha2 \\
\hline VNIII $M A T \alpha / \alpha$ & $\mathrm{C} 16$ & $\mathrm{~A} 1$ & $\mathrm{H} 1$ & E3 & ND & G16 & Ec10 & $\mathrm{Ha} 4$ \\
\hline
\end{tabular}

ND, Not done.

${ }^{\star}$ Distinct combination of letters and numbers represents different restriction profiles.

multigene studies (Boekhout et al., 2001; Lengeler et al., 2001; Meyer et al., 2003; Litvintseva et al., 2005a; Bovers et al., 2007, 2008b) have been needed until now for the precise identification of cryptococcal hybrids. However, in the current study, all three mating types $(\alpha / \mathbf{a}, \mathbf{a} / \alpha$ and $\alpha / \alpha)$ of the AD hybrids could be differentiated by either of the two RFLP approaches. They showed distinct restriction patterns from those of the other groups of the molecular and mating types. In spite of the similarity in patterns of $\mathrm{A} \alpha \mathrm{D} \alpha$ and $\mathrm{A} \alpha$ strains in the RFLP analysis of the CAP1 gene, an additional band was observed in $\mathrm{A} \alpha \mathrm{D} \alpha$ hybrids with EcoRII digestion following gel electrophoresis.

In contrast to the in silico analysis in which $\mathrm{AD}$ hybrids were analysed using a combination of molecular type VNI and VNIV alleles, $\mathrm{AaD} \alpha$ hybrids showed a mixed profile from the genotype VNB MATa profile rather than molecular type VNI MATa in the RFLP analysis of the $C A P 1$ gene (Fig. 6). The analysis showed that $\mathrm{A} \alpha \mathrm{D} \alpha$ hybrids displayed partial bands from the VNI MAT $\alpha$ profile rather than the VNII MAT $\alpha$ profile (Fig. 4), and A $\alpha$ a hybrids exhibited a mixed profile from VNI or VNII (both MAT $\alpha$ ) and VNIV MATa profiles (Fig. 2). In the RFLP analysis of the GEF1 gene, $\mathrm{A} \alpha \mathrm{Da}$ hybrids exhibited a combination of bands from the patterns of VNI MAT $\alpha$ and VNIV MATa, $\mathrm{A} \alpha \mathrm{D} \alpha$ hybrids exhibited a combination of bands from the

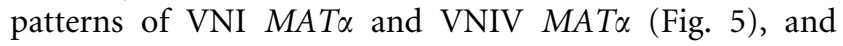
$\mathrm{AaD} \alpha$ hybrids exhibited a combination of bands from the patterns of VNI or VNB (both MATa) and VNIV MAT $\alpha$ (Fig. 3).

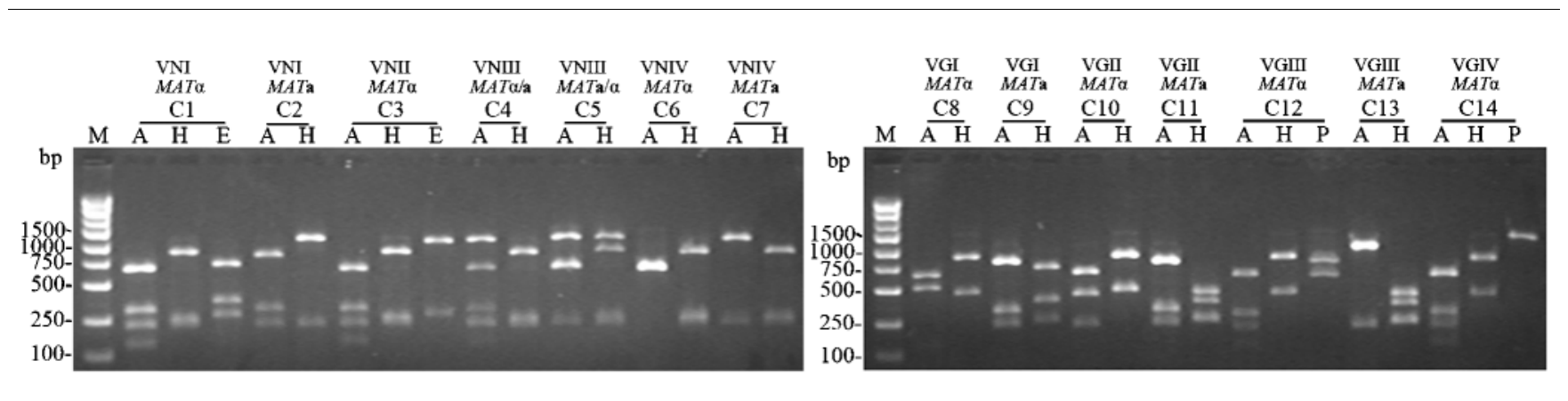

Fig. 2. RFLP patterns of $C A P 1$ gene fragments. Fourteen RFLP patterns $(C 1-C 14)$ were generated with the enzymes $A$ val $(A), H g i C l$ $(\mathrm{H})$, EcoRII (E) and Pstl (P). Left panel, RFLP patterns of C. neoformans; right panel, RFLP patterns of C. gattii. M, Molecular marker. 

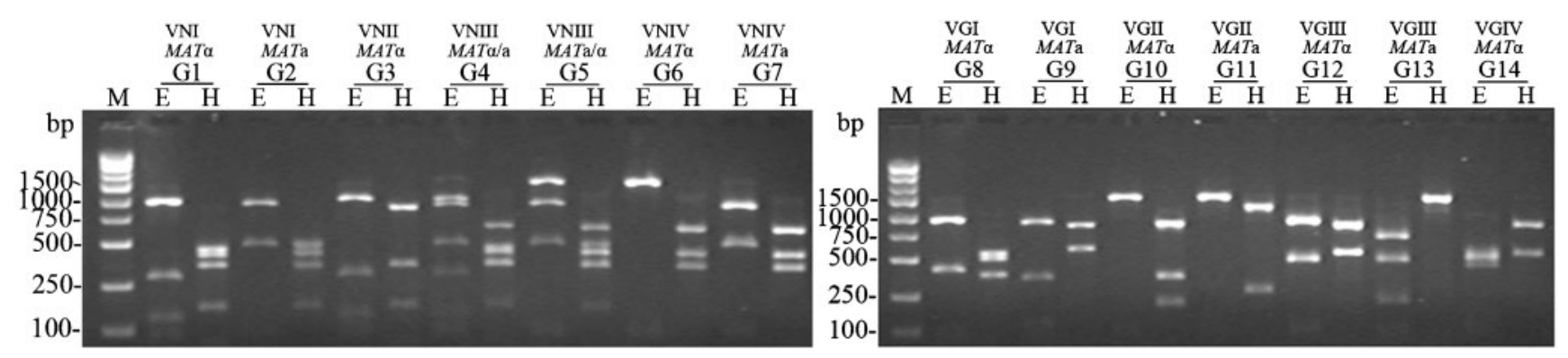

Fig. 3. RFLP patterns of GEF1 gene fragments. Fourteen RFLP patterns (G1-G14) were generated with the enzymes EcoT14I (E) and Hapll (H). Left panel, RFLP patterns of C. neoformans; right panel, RFLP patterns of C. gattii. M, Molecular marker.

A recent study suggested that many globally recovered $\mathrm{AaD} \alpha$ strains originated in sub-Saharan Africa because their MATa serotype A alleles were closely related to genotype VNB, which is geographically restricted to subSaharan Africa (Litvintseva et al., 2007). Likewise, the RFLP profile generated from the $\mathrm{AaD} \alpha$ strains tested here was found to contain bands from genotype VNB but not from molecular type VNI in the RFLP analysis of the CAP1 gene. The A $\alpha \mathrm{Da}$ strains exhibited a mixed profile of VNI MAT $\alpha$ and VNIV MATa in the RFLP analysis of the GEF1 gene. This hybrid genotype has also been detected by RFLP analysis of the $P L B 1$ gene and confirmed by phylogenetic analysis in previous studies (Latouche et al., 2003; Litvintseva et al., 2007), suggesting that the predominant distribution of the molecular type VNI may result in the detection of this genotype in many $\mathrm{A} \alpha \mathrm{Da}$ strains. Similarly, strains of $\mathrm{A} \alpha \mathrm{D} \alpha$, a progeny originated from same-sex mating, also had a MATa serotype A allele derived from molecular type VNI rather than VNII or VNB in accordance with other research (Litvintseva et al., 2007).

AD hybrids are diploid or aneuploid. Strains with loss of serotype A- or D-specific alleles in some loci, such as CLA4, LAC1, URA5 and IGS1, have been detected in several

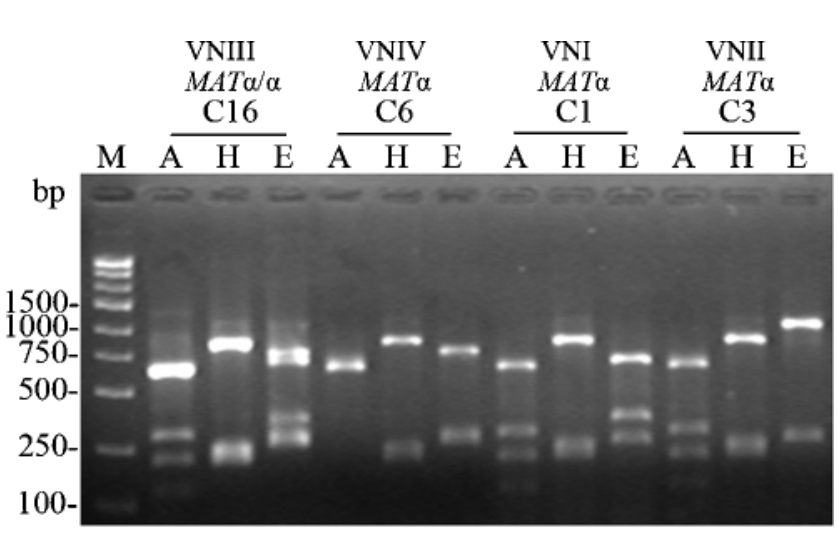

Fig. 4. RFLP patterns of CAP1 gene fragments. Pattern $\mathrm{C} 16$ $(\mathrm{A} \alpha \mathrm{D} \alpha)$ contains the bands from $\mathrm{C} 1$ (VNI MAT $\alpha)$ rather than $\mathrm{C} 3$

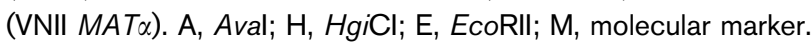

studies (Lengeler et al., 2001; Cogliati et al., 2006; Bovers et al., 2007). Just like the genes at the MAT locus, the absence of one mating-type allele was found in a few $\mathrm{AD}$ strains (less than $7 \%$ in $\mathrm{AD}$ hybrids) and a novel $\mathrm{AB}$ hybrid (Cogliati et al., 2001; Lengeler et al., 2001; Litvintseva et al., 2005a; Viviani et al., 2006; Bovers et al., 2008b). Because the MAT locus is inherited as a single unit (Lengeler et al., 2002), the absence of the CAP1 or GEF1 allele in the RFLP analysis indicates loss of the whole MAT locus.

In summary, for identification of the eight major molecular types of the Cryptococcus species complex, various molecular typing techniques have been developed such as PCR fingerprinting, amplified fragment length polymorphism analysis, RFLP, sequencing and luminex xMAP technology (Boekhout et al., 2001; Meyer et al., 2003; Diaz et al., 2005; Bovers et al., 2007), and the mating types are usually determined by separate PCR analyses or mating assays (Cogliati et al., 2001; Lengeler et al., 2001; Fraser et al., 2003; Campbell et al., 2005). In the present study, the molecular and mating types of all cryptococcal isolates tested were determined simultaneously by RFLP analysis based on either the CAP1 or the GEF1 gene. However, the RFLP analysis of the GEF1 gene seems to be more

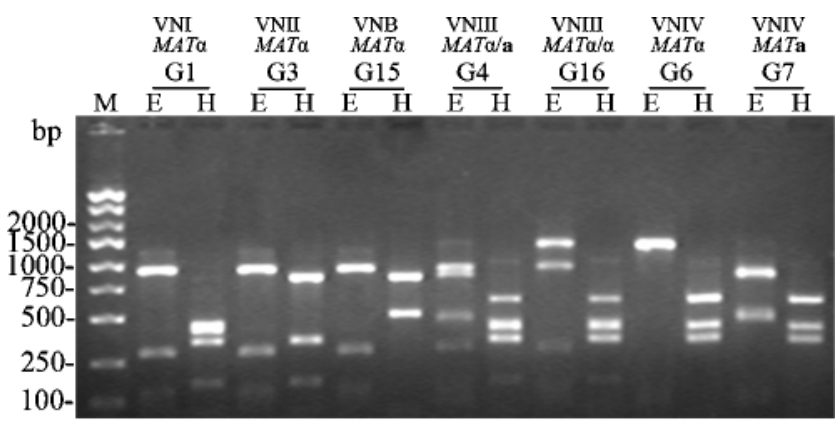

Fig. 5. RFLP patterns of GEF1 gene fragments. Pattern G4 $(\mathrm{A} \alpha \mathrm{Da})$ contains the bands from G7 (VNIV MATa) and G1 (VNI $M A T \alpha)$. Pattern $\mathrm{G} 16(\mathrm{~A} \alpha \mathrm{D} \alpha)$ contains the bands from $\mathrm{G} 6$ (VNIV

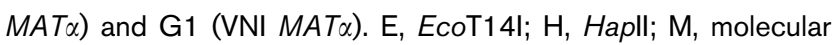
marker. 


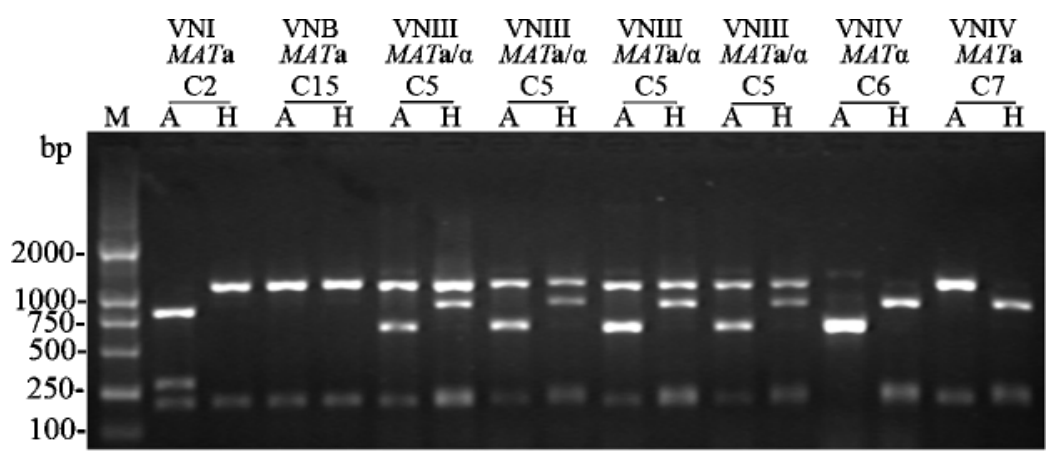

Fig. 6. RFLP patterns of CAP1 gene fragments. Pattern $\mathrm{C} 5(\mathrm{AaD} \alpha)$ contains the bands from C6 (VNIV MAT $\alpha$ ) and C15 (VNB MATa) rather than C2 (VNI MATa). A, Aval; $\mathrm{H}, \mathrm{HgiCl}$; $\mathrm{M}$, molecular marker.

convenient than the RFLP analysis of the CAP1 gene and should be a preferred typing method. Overall, the RFLP analysis developed here provides an alternative choice for easy identification of the molecular and mating types of strains belonging to the Cryptococcus species complex and may be a useful tool in clinical microbiological and epidemiological studies of this pathogenic yeast.

\section{ACKNOWLEDGEMENTS}

We thank Joseph Heitman, John R. Perfect, Anastasia P. Litvintseva, Wiley A. Schell, Sheryl Frank and Anna Floyd (Duke University Medical Center, Durham, USA), Wieland Meyer and Dee Carter (University of Sydney, Sydney, Australia), C. De Vroey (Prince Leopold Institut of Tropical Medicine, Belgium), Maria Anna Viviani (Istituto di Igiene e Medicina Preventiva, Milano, Italy), Kyung J. Kwon-Chung (National Institutes of Health, Bethesda, MD, USA), James W. Kronstad (University of British Columbia, Vancouver, BC, Canada), Teun Boekhout (Centraalbureau voor SchimmelculturesFungal Biodiversity Centre, Utrecht, The Netherlands), Jianping Xu (McMaster University, Hamilton, Canada) and Claudete R. Paula (University of São Paulo, São Paulo, Brazil) for strains. We also thank Wieland Meyer for his assistance in correction of the manuscript. This work was supported by a grant (no. 30870108) from the National Natural Science Foundation of China.

\section{REFERENCES}

Barchiesi, F., Cogliati, M., Esposto, M. C., Spreghini, E., Schimizzi, A. M., Wickes, B. L., Scalise, G. \& Viviani, M. A. (2005). Comparative analysis of pathogenicity of Cryptococcus neoformans serotypes A, D and $\mathrm{AD}$ in murine cryptococcosis. J Infect 51, 10-16.

Boekhout, T., Theelen, B., Diaz, M., Fell, J. W., Hop, W. C., Abeln, E. C., Dromer, F. \& Meyer, W. (2001). Hybrid genotypes in the pathogenic yeast Cryptococcus neoformans. Microbiology 147, 891-907.

Bovers, M., Hagen, F., Kuramae, E. E., Diaz, M. R., Spanjaard, L., Dromer, F., Hoogveld, H. L. \& Boekhout, T. (2006). Unique hybrids between the fungal pathogens Cryptococcus neoformans and Cryptococcus gattii. FEMS Yeast Res 6, 599-607.

Bovers, M., Diaz, M. R., Hagen, F., Spanjaard, L., Duim, B., Visser, C. E., Hoogveld, H. L., Scharringa, J., Hoepelman, I. M. \& other authors (2007). Identification of genotypically diverse Cryptococcus neoformans and Cryptococcus gattii isolates by Luminex xMAP technology. J Clin Microbiol 45, 1874-1883.

Bovers, M., Hagen, F., Kuramae, E. E. \& Boekhout, T. (2008a). Six monophyletic lineages identified within Cryptococcus neoformans and
Cryptococcus gattii by multi-locus sequence typing. Fungal Genet Biol 45, 400-421.

Bovers, M., Hagen, F., Kuramae, E. E., Hoogveld, H. L., Dromer, F., St-Germain, G. \& Boekhout, T. (2008b). AIDS patient death caused by novel Cryptococcus neoformans $\times$ C. gattii hybrid. Emerg Infect Dis 14, 1105-1108.

Bui, T., Lin, X., Malik, R., Heitman, J. \& Carter, D. (2008). Isolates of Cryptococcus neoformans from infected animals reveal genetic exchange in unisexual, $\alpha$ mating type populations. Eukaryot Cell, (in press) doi:10.1128/EC.00097-08

Campbell, L. T., Fraser, J. A., Nichols, C. B., Dietrich, F. S., Carter, D. \& Heitman, J. (2005). Clinical and environmental isolates of Cryptococcus gattii from Australia that retain sexual fecundity. Eukaryot Cell 4, 1410-1419.

Casadevall, A. \& Perfect, J. R. (1998). Cryptococcus neoformans. Washington, DC: American Society for Microbiology.

Cogliati, M., Esposto, M. C., Clarke, D. L., Wickes, B. L. \& Viviani, M. A. (2001). Origin of Cryptococcus neoformans var. neoformans diploid strains. J Clin Microbiol 39, 3889-3894.

Cogliati, M., Esposto, M. C., Tortorano, A. M. \& Viviani, M. A. (2006). Cryptococcus neoformans population includes hybrid strains homozygous at mating-type locus. FEMS Yeast Res 6, 608-613.

Diaz, M. R., Boekhout, T., Kiesling, T. \& Fell, J. W. (2005). Comparative analysis of the intergenic spacer regions and population structure of the species complex of the pathogenic yeast Cryptococcus neoformans. FEMS Yeast Res 5, 1129-1140.

Fraser, J. A., Subaran, R. L., Nichols, C. B. \& Heitman, J. (2003). Recapitulation of the sexual cycle of the primary fungal pathogen Cryptococcus neoformans var. gattii: implications for an outbreak on Vancouver Island, Canada. Eukaryot Cell 2, 1036-1045.

Fraser, J. A., Giles, S. S., Wenink, E. C., Geunes-Boyer, S. G., Wright, J. R., Diezmann, S., Allen, A., Stajich, J. E., Dietrich, F. S. \& other authors (2005). Same-sex mating and the origin of the Vancouver Island Cryptococcus gattii outbreak. Nature 437, 13601364.

Keller, S. M., Viviani, M. A., Esposto, M. C., Cogliati, M. \& Wickes, B. L. (2003). Molecular and genetic characterization of a serotype A MATa Cryptococcus neoformans isolate. Microbiology 149, 131-142.

Kidd, S. E., Hagen, F., Tscharke, R. L., Huynh, M., Bartlett, K. H., Fyfe, M., Macdougall, L., Boekhout, T., Kwon-Chung, K. J. \& Meyer, W. (2004). A rare genotype of Cryptococcus gattii caused the cryptococcosis outbreak on Vancouver Island (British Columbia, Canada). Proc Natl Acad Sci U S A 101, 17258-17263.

Kidd, S. E., Guo, H., Bartlett, K. H., Xu, J. \& Kronstad, J. W. (2005). Comparative gene genealogies indicate that two clonal lineages of Cryptococcus gattii in British Columbia resemble strains from other geographical areas. Eukaryot Cell 4, 1629-1638. 
Kwon-Chung, K. J. \& Varma, A. (2006). Do major species concepts support one, two or more species within Cryptococcus neoformans? FEMS Yeast Res 6, 574-587.

Kwon-Chung, K. J., Edman, J. C. \& Wickes, B. L. (1992). Genetic association of mating types and virulence in Cryptococcus neoformans. Infect Immun 60, 602-605.

Kwon-Chung, K. J., Boekhout, T., Fell, J. W. \& Diaz, M. (2002). Proposal to conserve the name Cryptococcus gattii against $C$. hondurianus and C. bacillisporus (Basidiomycota, Hymenomycetes, Tremellomycetidae). Taxon 51, 804-806.

Latouche, G. N., Huynh, M., Sorrell, T. C. \& Meyer, W. (2003). PCRrestriction fragment length polymorphism analysis of the phospholipase B (PLB1) gene for subtyping of Cryptococcus neoformans isolates. Appl Environ Microbiol 69, 2080-2086.

Lengeler, K. B., Wang, P., Cox, G. M., Perfect, J. R. \& Heitman, J. (2000). Identification of the MATa mating-type locus of Cryptococcus neoformans reveals a serotype A MATa strain thought to have been extinct. Proc Natl Acad Sci U S A 97, 14455-14460.

Lengeler, K. B., Cox, G. M. \& Heitman, J. (2001). Serotype AD strains of Cryptococcus neoformans are diploid or aneuploid and are heterozygous at the mating-type locus. Infect Immun 69, 115-122.

Lengeler, K. B., Fox, D. S., Fraser, J. A., Allen, A., Forrester, K., Dietrich, F. S. \& Heitman, J. (2002). Mating-type locus of Cryptococcus neoformans: a step in the evolution of sex chromosomes. Eukaryot Cell 1, 704-718.

Lin, X., Litvintseva, A. P., Nielsen, K., Patel, S., Floyd, A., Mitchell, T. G. \& Heitman, J. (2007). $\alpha \mathrm{AD} \alpha$ hybrids of Cryptococcus neoformans: evidence of same-sex mating in nature and hybrid fitness. PLoS Genet 3, 1975-1990.

Litvintseva, A. P., Kestenbaum, L., Vilgalys, R. \& Mitchell, T. G. (2005a). Comparative analysis of environmental and clinical populations of Cryptococcus neoformans. J Clin Microbiol 43, 556-564.

Litvintseva, A. P., Thakur, R., Reller, L. B. \& Mitchell, T. G. (2005b). Prevalence of clinical isolates of Cryptococcus gattii serotype C among patients with AIDS in sub-Saharan Africa. J Infect Dis 192, 888-892.

Litvintseva, A. P., Thakur, R., Vilgalys, R. \& Mitchell, T. G. (2006). Multilocus sequence typing reveals three genetic subpopulations of Cryptococcus neoformans var. grubii (serotype A), including a unique population in Botswana. Genetics 172, 2223-2238.

Litvintseva, A. P., Lin, X., Templeton, I., Heitman, J. \& Mitchell, T. G. (2007). Many globally isolated AD hybrid strains of Cryptococcus neoformans originated in Africa. PLoS Pathog 3, e114.

Meyer, W., Castañeda, A., Jackson, S., Huynh, M., Castañeda, E. \& the IberoAmerican Cryptococcal Study Group (2003). Molecular typing of IberoAmerican Cryptococcus neoformans isolates. Emerg Infect Dis 9, 189-195.

Mitchell, T. G. \& Perfect, J. R. (1995). Cryptococcosis in the era of AIDS - 100 years after the discovery of Cryptococcus neoformans. Clin Microbiol Rev 8, 515-548.

Speed, B. \& Dunt, D. (1995). Clinical and host differences between infections with the two varieties of Cryptococcus neoformans. Clin Infect Dis 21, 28-36.

Tortorano, A. M., Viviani, M. A., Rigoni, A. L., Cogliati, M., Roverselli, A. \& Pagano, A. (1997). Prevalence of serotype D in Cryptococcus neoformans isolates from HIV positive and HIV negative patients in Italy. Mycoses 40, 297-302.

Trilles, L., Fernández-Torres, B., Lazéra Mdos, S., Wanke, B. \& Guarro, J. (2004). In vitro antifungal susceptibility of Cryptococcus gattii. J Clin Microbiol 42, 4815-4817.

Viviani, M. A., Cogliati, M., Esposto, M. C., Lemmer, K., Tintelnot, K., Colom Valiente, M. F., Swinne, D., Velegraki, A., Velho, R. \& the European Confederation of Medical Mycology (ECMM) Cryptococcosis Working Group (2006). Molecular analysis of 311 Cryptococcus neoformans isolates from a 30-month ECMM survey of cryptococcosis in Europe. FEMS Yeast Res 6, 614-619. 